\title{
Periodontal Treatment by Dentistry Undergraduate Students. Assessment of the Patient's Oral Quality of Life: A Prospective Pilot Study.
}

\section{María Paloma Alvarez Azaustre}

Universidad Europea de Valencia

\section{Manuel Bravo}

Universidad de Granada Facultad de Odontologia

Antonio Magan-Fernandez ( $\square$ amaganf@ugr.es )

Universidad de Granada https://orcid.org/0000-0001-6430-2276

Alberto Rodríguez-Archilla

Universidad de Granada Facultad de Odontologia

\section{Carmen Llena}

Universitat de Valencia Facultat de Medicina i Odontologia

\section{Francisco Mesa}

Universidad de Granada Facultad de Odontologia

\section{Research article}

Keywords: Periodontal debridement, Periodontitis, Students, Oral health, Quality of life.

Posted Date: September 9th, 2020

DOl: https://doi.org/10.21203/rs.3.rs-52299/v1

License: (1) This work is licensed under a Creative Commons Attribution 4.0 International License. Read Full License 


\section{Abstract}

Background: Our aim was to assess the impact of non-surgical periodontal treatment on oral healthrelated quality of life of patients with periodontitis, performed by undergraduate dental students.

Methods: An observational prospective one cohort study pre-post test was performed, involving 31 undergraduate dental students. A complete periodontal examination was performed before and after receiving non-surgical periodontal treatment. The main independent clinical variables assessed were the grade of periodontal inflammation and the number of teeth with periodontitis. Oral health-related quality of life was assessed before and after treatment through the Oral Impacts on Daily Performances (OIDP) questionnaire. The association between the extension of periodontal treatment (measured as number of treated teeth) and final OIDP score was assessed, adjusting for age, sex and baseline OIDP, in a multiple linear regression model.

Results: 34 patients were enrolled and treated by the undergraduate students. The global absolute score, the mean OIDP value, was reduced from 26.2 to 12 . The global percent score, percentage of impact, was reduced from 13 to 6 . Non-surgical periodontal treatment showed an improvement in oral health-related quality of life. However, no association between the number of treated teeth and none of the aspects measured by ODIP was observed post-treatment, adjusting for age, sex and baseline OIDP.

Conclusion: Non-surgical periodontal treatment performed by undergraduate dental students on periodontal patients, did not show any impact on oral health-related quality of life, measured by OIDP.

\section{Background}

Diagnosis, instructions in oral hygiene and non-surgical treatment with debridement of the surface of the dental root, are periodontal competences and skills that the undergraduate Dentistry student must acquire.(1) In a recent study through self-administered questionnaires performed in dental students from the USA, $63.1 \%$ of them judged the periodontal care that they provided to their patients as inadequate.(2) Authors have described several factors to explain these results, such as treatment provided by multiple students over time, supervision by a single student, academic requirements that limit clinical time and prevents the reevaluation of the patient, and the non-compliance to the appointments by the patient to complete the treatment. Albaraki et al. showed that only $3 \%$ of male patients and $4.5 \%$ of female patients attended to the first periodontal re-evaluation by undergraduate students from Riyadh Elm University in Riyadh, Saudi Arabia.(3)

Knowing the opinion of the patient would be an important aspect of the student-patient relationship, since the assessment of the non-surgical periodontal treatment by the patient could be an important part of the learning procedure of the undergraduate student. This could be performed by assessing the satisfaction and/or oral health-related quality of life (OHRQoL) of the patients, through the implementation of a questionnaire before and after treatment aiming to be assessed. To our knowledge, there is no scientific evidence about this topic. 
The Oral Health Status and Oral Impacts on Daily Performances (OIDP) survey for adults, was developed and validated in English by Adulyanon and Sheiham, was presented in the Chapel Hill Conference of 1996,(4) and is focused on measuring severe oral impairments affecting a person's ability to perform daily activities. It includes 8 daily life aspects such as eating and enjoying food, speaking and pronouncing well, difficulty brushing teeth or using mouth rinse, loss of emotional stability (angry or irritated) due to oral cause, difficulty resting or sleeping well, embarrassed by teeth appearance, working and contact with people. OIDP index is a relevant tool to assess oral health problems in a specific sociocultural community (5) and was validated for adults in Spain by Montero et al. in 2008.(6)

The objective of our study was to assess the impact on OHRQoL, measured by OIDP index, of nonsurgical periodontal treatment of patients with periodontitis treated by undergraduate dental students at the University of Granada (Spain).

\section{Methods}

\section{Study Design}

The study protocol received the approval of the Ethics Committee of Human Research of the University of Granada (Ref. 1138/CEIH/2020). An observational prospective one cohort study pre-post test was performed, involving undergraduate dental students from the School of Dentistry of the University of Granada (Spain), in the first semester of academic year 2019-2020. All patients were referred to the Periodontology service of the School of Dentistry and underwent nonsurgical periodontal treatment.

Inclusion criteria were age $>18$ years with diagnosis of periodontitis according to the following criteria: presence of at least 2 sites with pocket probing depth (PPD) $\geq 4 \mathrm{~mm}$ at 2 different teeth, clinical attachment loss (CAL) $\geq 3 \mathrm{~mm}$, with bleeding on probing (BOP) and bone loss $\geq 2 \mathrm{~mm}$ confirmed by orthopantomography.(7) Exclusion criteria were antibiotic and/or anti-inflammatory therapy 3 months before the study, previous history of periodontal therapy in the last year, ischemic cardiopathy, anticoagulated patients, hepatitis or HIV-positive patients, or diagnosis of any psychological disorder that could interfere in the comprehension of the questionnaire. The number of present teeth was not considered as an exclusion criterion. Informed consent was obtained from all participants. This manuscript was prepared according to the Strengthening the Reporting of Observational Studies in Epidemiology (STROBE) guidelines.(8)

\section{Sample size}

This pilot study was designed to study the association between scaling and root planing (\# of treated teeth) and OIDP after treatment. We decided to have at least 25 patients to detect a large effect size $(r=0.5$, i.e., the association is measured by correlation) according to Cohen,(9) with alpha $=0.05$ and power=0.8. Finally, 34 patients were enrolled in the study.

\section{Periodontal examination and treatment}


All diagnostic exams and treatments were performed by undergraduate students. A complete periodontal examination was performed on each patient, gathering the following periodontal variables in a periodontal chart: PPD, CAL, BOP,(10) Plaque Index,(11) gingival recession and number of periodontal teeth. The exam was performed with PCP-UNC 15 (Hu-Friedy, Chicago, IL, USA) manual periodontal probe.

All patients received non-surgical periodontal treatment, which consisted of oral hygiene instructions, supragingival plaque control and subgingival scaling and root planning (SRP). In the first appointment, clinical examination and supragingival calculus elimination by ultrasonic device were performed (Woodpecker G1 tips, mod. HW-3H, Guilin, China). The second appointment was programmed in the following week, and dental students performed SRP per quadrant, under local anesthesia, with Manual periodontal Gracey curettes 5/6, 7/8, 11/12, 13/14 (Hu-Friedy, Chicago, IL, USA) to eliminate calculus and subgingival biofilm. Maintenance appointments were programmed 4-6 weeks after treatment, with a periodontal examination and reassessment of initial periodontal parameters, performed by the same student.

\section{OIDP Questionnaire}

The OIDP questionnaire, validated for adults in Spain by Montero et al in 2008,(6) was administered to each patient before treatment and at the 4-6 weeks appointment. All students received previous training in OHRQoL and specific characteristics, usage and interpretation of the OIDP survey, as well as the procedure to perform the interview and administer the survey, regarding data gathering and bias prevention. This training was done through a specific seminar by one of the researchers participating in the study, with previous experience in the topic, and the supervision of a professor was provided while collecting first survey data. The survey was interview-administered by each student to its corresponding patient.

The questionnaire consisted of 8 structured aspects asked to the patient, regarding 8 dimensions or activities in daily life (eating, speaking, cleaning teeth, working, social relation, sleeping/relaxing, smiling and emotional status). First, the patient answered on a dichotomous format (yes/no) if each dimension has been affected in a previously-established period of time ( 6 months for the baseline evaluation and 1 month -i.e. for the post-treatment evaluation). If any answer was "yes", the frequency was assessed by the following score: 1 (less than 1 time/month), 2 (1-2 times/month), 3 (1-2 times/week), 4 (3-4 times/week) and 5 (almost every day). If the frequency affected a certain period of time, a different scoring was applied: 1 (< 5 days), 2 (> 5 days), 3 (> 1 month), 4 (>3 months) and 5 (the whole period). Likert scale was then applied to assess "severity" from 0 (null effect) to 5 (very severe effect).(6) The rating scale on each dimension was between 0 (zero) and 25 . The total score of the patient, was obtained by multiplying frequency by severity on each dimension, and adding up the individual values of all dimensions. Maximum score of the questionnaire was 200. Impact index was obtained by the formula (total score $\times 200$ )/100. Individual rating was therefore expressed as a percentage of impact. The higher the percentage of impact, the lower the OHRQoL they had and vice versa. 
All data regarding the survey, were collected hand written by the students, and then converted to excel sheets that calculated the total score of each patient and individual OIDP impact index on OHRQoL.

\section{Statistical analysis}

Statistical analysis was performed using SPSS 20 (IBM Corp., Armonk, NY, USA). Statistical tests used are described in table footnotes. To assess the effect of periodontal treatment, the pre-post OIDP comparison was not considered to be the most adequate approach, due to the absence of control group and also to the fact that the questionnaire was administered by dental students that are being supervised, and that a bond student-patient is formed that may alter (improve) the answers while administering the post-treatment OIDP questionnaire. Therefore, we analyzed the possible association between the extension of periodontal treatment (measured as number of treated periodontal teeth) and final OIDP score, adjusted for age, sex, and baseline OIDP score, by multiple linear regression. If the periodontal treatment is the cause of the improvement in OHRQoL, a dose-response relationship would be expected (between number of treated teeth and final OIDP score).

\section{Results}

The study included 34 patients, 18 men (52.9\%) and 16 women (47.1\%), with a mean age of $52.6 \pm 11.1$ (mean \pm standard deviation). Age groups and baseline values of the two independent clinical variables included in the study (BOP and number of SRP treated teeth) are described in Table 1. 
Table 1

Patient's characteristics at baseline $(n=34)$.

\begin{tabular}{|ll|}
\hline Variable & $\mathbf{n}(\%)$ \\
\hline Age (yrs.) & $10(29.4)$ \\
$50-49$ & $12(35.3)$ \\
$60-71$ & $12(35.3)$ \\
mean \pm sd & $52.6 \pm 11.1$ \\
Sex & \\
Male & $18(52.9)$ \\
Female & $16(47.1)$ \\
\hline BOP (\%) & \\
$4-33$ & $7(20.6)$ \\
$34-66$ & $17(50.0)$ \\
$67-87$ & $10(29.4)$ \\
mean \pm sd & $53 \pm 24$ \\
SRP (\# Treated teeth) & \\
$5-14$ & $12(35.3)$ \\
$15-19$ & $11(32.4)$ \\
$20-28$ & $11(32.4)$ \\
mean \pm sd & $16.8 \pm 5.3$ \\
BOP: Bleeding on Probing. SRP: Scaling and Root Planing \\
\hline
\end{tabular}

Table 2 describes OIDP score in both baseline and after periodontal treatment. The percentage impact at baseline (referred to the previous 6 months) is 13\%, compared to the one after treatment (referred to the previous 4-6 weeks) of $6 \%$. Also, Table 2 shows that the prevalence of impact, referred to the number of affected dimensions, was of $2.35 \pm 1.77$ at baseline, and of $1.35 \pm 1.59$ after treatment. Therefore, a greater number of OIDP dimensions were affected at baseline compared to the post-treatment examination. The global absolute score, the mean OIDP score, changed from $26.2 \pm 35.6$ to $12 \pm 31.2$, and the global percent score, percentage of the impact, from $13 \pm 18 \%$ to $6 \pm 16 \%$. Therefore, an improvement in OHRQoL was observed in the patients. Considering dimensions, the most affected ones before treatment in order of relevance were teeth brushing, eating and smiling. After the treatment the most affected ones were smiling, eating, talking and teeth brushing. 
Table 2

OIDP score (mean \pm sd) in the patients of the study $(n=34) \dagger$

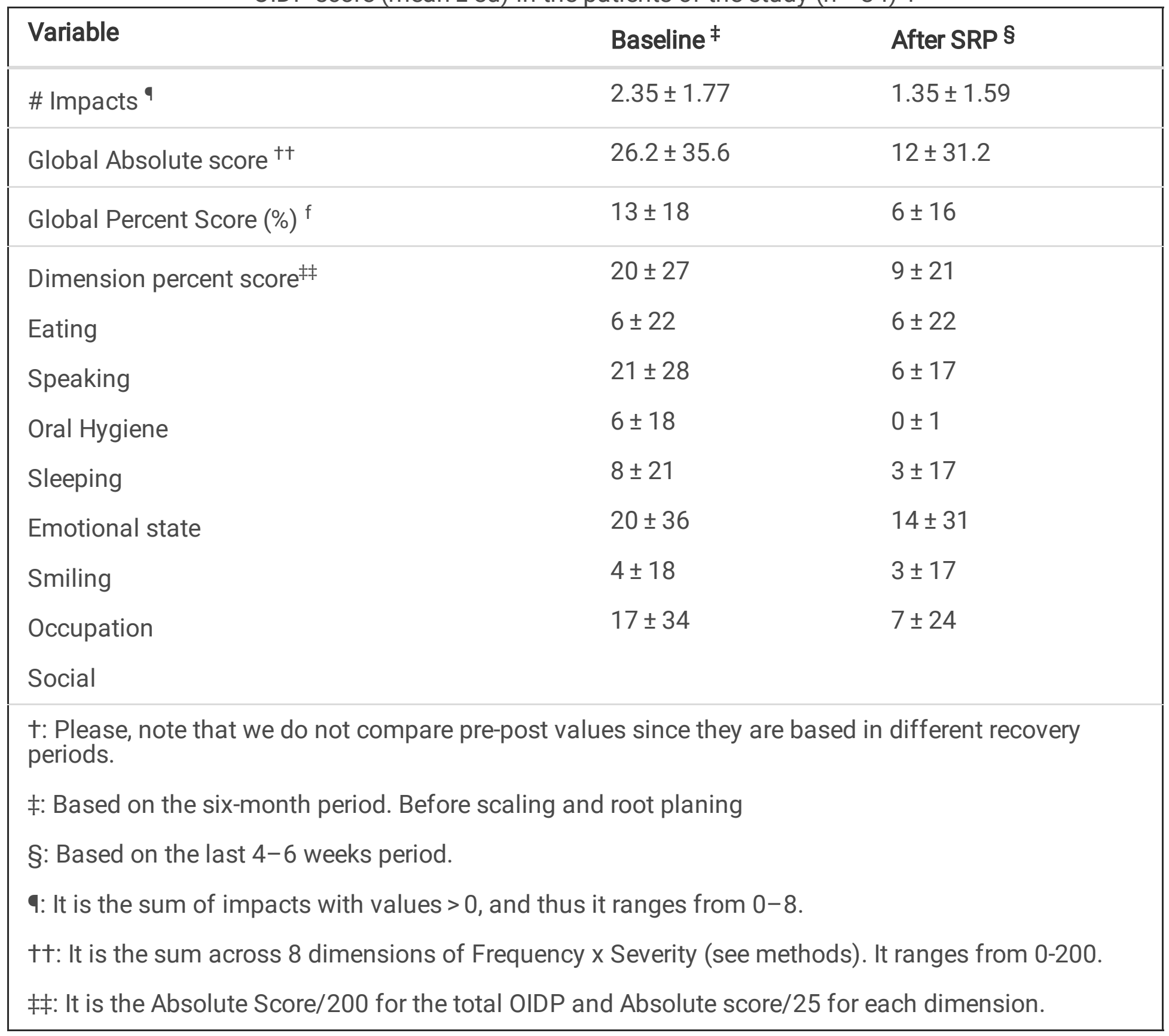

There was no statistically significant association, measured by Pearson's correlation analysis, between BOP or number of treated teeth (which is a proxy variable to extension of periodontal status) with OIDP, measured as number of impacts, or absolute or percent scores (results not shown).

Table 3 shows the association, adjusted by age, sex and baseline OIDP values, between the number of treated teeth and OIDP score post-treatment. No significant association was found between number of teeth treated and the improvement in OHRQoL, although the coefficient of determination $\left(\mathrm{R}^{2}\right)$ of all models are slightly high, but it is derived from the association with baseline OIDP values. Although clinical evaluation is not the purpose of this study, it should be noted that the study was supervised by 4 
professors and the periodontal chart performed post-treatment showed that periodontal disease was controlled, and all patients entered in supportive periodontal therapy (results not shown).

Table 3

Multiple linear regression models of factors associated with OIDP + after SRP $(n=34)$

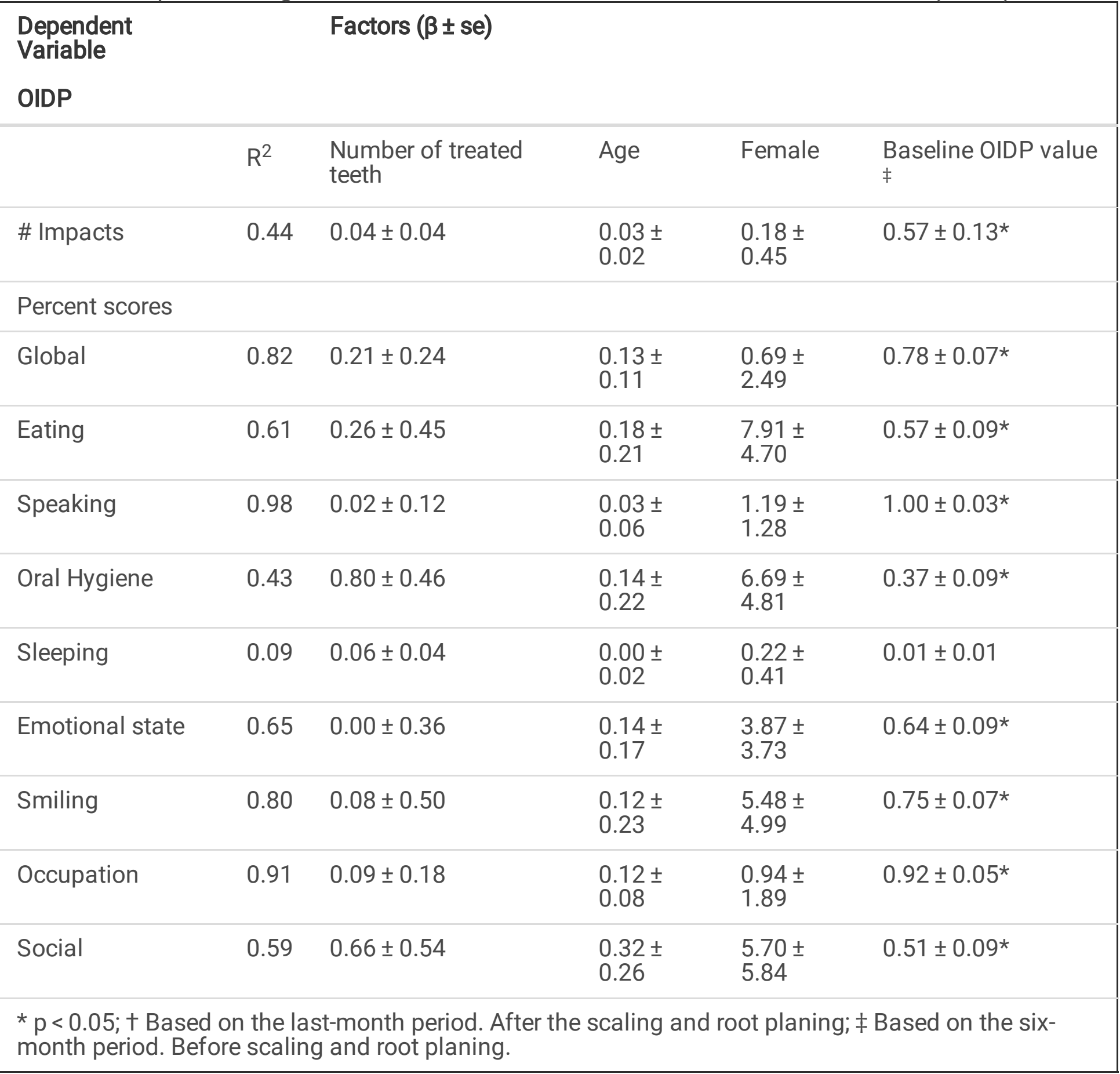

\section{Discussion}

Periodontal treatment performed by undergraduate Dentistry students was not associated with an improvement in OHRQoL, assessed by OIDP score. Although there was an improvement in the OIDP parameters after periodontal treatment (Table 2), this improvement was not due to treatment, as 
demonstrated by the lack of a statistically significant association between the number of teeth treated and final OIDP.

OIDP survey, which has been widely tested in different contexts, has been validated for the Spanish adult population,(6) as well as in children and adolescents.(12) Regarding our results, it seems that it is not a sensitive approach to evaluate periodontal status both at baseline (Pearson's correlation, results not shown) and after periodontal treatment. Multivariate linear regression analysis showed that there was no association between the number of treated teeth, a variable of the intensity of the therapy, and the improvement in OHRQoL in the 8 dimensions evaluated by the OIDP survey. These results could be explained for several reasons. The presence of an information bias, in which the patients did not want to give negative answers to the students at the post-treatment revision, when asked about the improvement in the different aspects. The different time frame of both surveys, the first one covering the previous 6 months and the second one the previous 4-6 weeks, or the small sample size, where the periodontal condition is not correctly perceived by the patient and showed with this type of surveys.

In the present study, the OIDP questionnaire was administered as a personal interview, in which each student interviewed his patient. The students previously received an explanatory session of the components and terms of the questionnaire and were trained its administration between themselves. This procedure allowed students to learn this methodology of studies on OHRQoL, and ensured that all the questionnaires were correctly performed, avoiding any loss to follow-up (in our study, all patients completed both surveys). However, it could be possible that the way of answering of each patient might be influenced by the way of asking of each student.

Segura et al.,(13) reviewed the most used instruments to assess the impact of periodontitis on OHRQoL, and found that the most widely used questionnaire was the Oral Health Impact Profile (OHIP-14), followed by others, among which OIDP is included. Both questionnaires are similar, the first uses a Likerttype scale to measure the negative effects on the performance on daily activities in the last twelve months in seven dimensions. These dimensions are: functional limitation, physical pain, psychological distress, physical disability, psychological disability, social disability, handicaps. The OIDP, also uses a Likert-type scale to measure the negative impact on basic daily activities during the last six months. These 8 dimensions are: eating, speaking, hygiene, occupational activities, social relations, sleepingrelaxing, smiling, and emotional state. Both instruments have good psychometric properties and their strengths could be complementary in assessing the impact of oral health on quality of life.(14) In the present study, OIDP score was chosen, as it had been validated for Spain by members of our group.

A recent systematic review aimed to verify whether oral conditions (tooth loss, periodontal disease, dental caries) were negatively associated with health-related quality of life (HRQoL) in adults. Four out of seven studies reported that periodontal disease impairs HRQoL, and 1 study showed that periodontal disease is positively associated with HRQoL. Authors concluded that mixed and inconclusive findings were observed for the association between periodontal disease and HRQoL.(15) OIDP score has been used to assess quality of life in relation to periodontal health in several studies. Wandera et al. used OIDP to 
assess the impact on periodontal status, determined by the periodontal community index, on pregnant women. They concluded that OIDP showed discriminative validity in identifying women with clinical evidence of tooth loss, but was less convincing in identifying women with clinically defined periodontal disease.(16)

Santuchi et al. compared the effect on the OHRQoL, using the OIDP questionnaire, of two protocols of non-surgical periodontal treatment: SRP by quadrants and Full mouth Disinfection (FMD) in one single session. Both protocols showed an improvement in OHRQoL, with no differences between treatment approaches.(17) The students in our center followed the SRP modality by quadrants for the non-surgical periodontal treatment, since scientific evidence showed that FMD approach does not provide any additional benefit.(18) Costa et al. recently evaluate the effects of compliance during periodontal supportive therapy in the OIDP scores, and showed that patients who are compliant presented lower OIDP scores compared to the irregular compliers.(19) The same group published in 2011 higher scores of neuroticism and conscientiousness $\left(\mathrm{R}^{2}=68 \% ; p<0.001\right)$ associated with higher OIDP scores among periodontal maintenance compliers patients.(20)

To our knowledge, this study shows for the first time in dental students that periodontal treatment, being part of their learning, can be clinically effective, although its impact on the OHRQoL of these patients could not be detected by OIDP scoring. Both correlations of the OIDP scores with the two initial clinical variables at baseline, as well as the correlation between the OIDP results after treatment and the number of teeth that received periodontal treatment, support this. The absence of a trend or gradient with any of the OIDP dimensions in the linear regression model also supports this finding.

Limitations of this pilot study, are mainly the small sample size and the absence of a control group. For ethical reasons, a control group could have been enrolled and these patients could have been treated at another time, but academic requirements such as limited clinical time, did not allow this design. The fact that all the surveys were not performed by the same interviewer, is another inherent limitation to the university environment in which the study was performed.

\section{Conclusions}

The conclusion of this study is that non-surgical periodontal treatment performed by undergraduate dental students at the University of Granada (Spain), had no impact on the OHRQoL perceived by the patients, measured by OIDP.

\section{List Of Abbreviations}

OHRQoL: Oral health-related quality of life.

OIDP: Oral Impacts on Daily Performances.

PPD: Pocket probing depth. 
CAL: Clinical attachment loss.

BOP: Bleeding on probing.

SRP: Scaling and root planning.

STROBE: Strengthening the Reporting of Observational Studies in Epidemiology.

OHIP-14: Oral Health Impact Profile.

HRQoL: Health-related quality of life.

FMD: Full mouth Disinfection.

\section{Declarations}

Ethics approval and consent to participate: The study protocol received the approval of the Ethics Committee of Human Research of the University of Granada (Ref. 1138/CEIH/2020). Written informed consent was obtained from all participants.

Consent for publication: Not applicable.

Availability of data and materials: The datasets used and/or analysed during the current study are available from the corresponding author on reasonable request.

Competing interests: The authors declare that they have no competing interests.

Funding: Not applicable.

Authors' contributions: FM and MPAA were involved in the Conception and designed the work. Data acquisition was performed by MPAA and ARA, and the analysis was performed by MB. Draft of the manuscript was performed by AMD and CLL. All authors read and approved the submitted version of the manuscript.

Acknowledgements: This study was supported Research Group \#CTS 583 (Junta de Andalucía, Spain).

\section{References}

1. European Federation of Periodontology. Undergraduate Education - Curricular Guidelines. https://www.efp.org/education/undergraduate/ Accesed March 30, 2020.

2. Chandrasekaran S, Powell C, De la Rosa L, Mittal A, Johnson L. Dental Students' Reflections on Quality of Periodontal Care in Dental School Clinics. J Dent Educ. 2017;81(1):14-21.

3. Albaraki A, Alghamdi M, Alghamdi F, Alduhaimi M, Alaskar M, Shaheen RS. Frequency of Periodontal Therapy Re-evaluation and Maintenance by the Students at Riyadh Elm University, Saudi Arabia - A 
Retrospective Study. EC Dental Science. 2019;18(8):1830-5.

4. Adulyanon S, Sheiham A. Oral impacts on daily performances. In: Slade GD, editor. Measuring oral health and quality of life. Chapel Hill: University of North Carolina; 1997. p. 151-60.

5. Sombateyotha K, Mahaweerawat U. Oral Health Status and Oral Impact on Daily Performance in Elderly in Northeastern Region Thailand. European Journal of Sustainable Development. 2017;6(2):240.

6. Montero J, Bravo M, Albaladejo A. Validation of two complementary oral-health related quality of life indicators (OIDP and OSS 0-10) in two qualitatively distinct samples of the Spanish population. Health Qual Life Outcomes. 2008;6:101.

7. Locker D. Measuring oral health: a conceptual framework. Community Dent Health. 1988;5(1):3-18.

8. von Elm E, Altman DG, Egger M, Pocock SJ, Gotzsche PC, Vandenbroucke JP, et al. The Strengthening the Reporting of Observational Studies in Epidemiology (STROBE) statement: guidelines for reporting observational studies. J Clin Epidemiol. 2008;61(4):344-9.

9. Cohen J. Statistical power analysis for the behavioural sciences. $2^{\circ} \mathrm{Ed}$. ed. New Jersey: Lawrence Erlbaum Associates; 1988.

10. Ainamo J, Bay I. Problems and proposals for recording gingivitis and plaque. Int Dent J. 1975;25(4):229-35.

11. O'Leary TJ, Drake RB, Naylor JE. The plaque control record. J Periodontol. 1972;43(1):38.

12. Cortes-Martinicorena FJ, Rosel-Gallardo E, Artazcoz-Oses J, Bravo M, Tsakos G. Adaptation and validation for Spain of the Child-Oral Impact on Daily Performance (C-OIDP) for use with adolescents. Med Oral Patol Oral Cir Bucal. 2010;15(1):e106-11.

13. Segura Cardona A, De La Hoz RP. Instrumentos para medir la calidad de vida relacionada con la salud oral: una revisión sistemática. Revista Salud Uninorte. 2017;33:504-16.

14. Montero J, Lopez JF, Vicente MP, Galindo MP, Albaladejo A, Bravo M. Comparative validity of the OIDP and OHIP-14 in describing the impact of oral health on quality of life in a cross-sectional study performed in Spanish adults. Med Oral Patol Oral Cir Bucal. 2011;16(6):e816-21.

15. Haag DG, Peres KG, Balasubramanian M, Brennan DS. Oral Conditions and Health-Related Quality of Life: A Systematic Review. J Dent Res. 2017;96(8):864-74.

16. Wandera MN, Engebretsen IM, Rwenyonyi CM, Tumwine J, Astrom AN, Group P-ES. Periodontal status, tooth loss and self-reported periodontal problems effects on oral impacts on daily performances, OIDP, in pregnant women in Uganda: a cross-sectional study. Health Qual Life Outcomes. 2009;7:89.

17. Santuchi CC, Cortelli JR, Cortelli SC, Cota LO, Fonseca DC, Alencar CO, et al. Scaling and Root Planing per Quadrant Versus One-Stage Full-Mouth Disinfection: Assessment of the Impact of Chronic Periodontitis Treatment on Quality of Life-A Clinical Randomized, Controlled Trial. J Periodontol. 2016;87(2):114-23. 
18. Eberhard J, Jepsen S, Jervoe-Storm PM, Needleman I, Worthington HV. Full-mouth treatment modalities (within 24 hours) for chronic periodontitis in adults. Cochrane Database Syst Rev. 2015(4):CD004622.

19. Costa FO, Cortelli SC, Costa AA, Cyrino RM, Cortelli JR, Miranda Cota LO. Impact of compliance during periodontal maintenance therapy on oral health-related quality of life: A 6-year follow-up. J Dent. 2019;83:50-5.

20. Costa FO, Miranda Cota LO, Pereira Lages EJ, Vilela Camara GC, Cortelli SC, Cortelli JR, et al. Oral impact on daily performance, personality traits, and compliance in periodontal maintenance therapy. J Periodontol. 2011;82(8):1146-54.

\section{Supplementary Files}

This is a list of supplementary files associated with this preprint. Click to download.

- EnglishOIDPQuestionnaire.docx

- STROBEChecklist.docx 